06

\title{
Динамика волнового пакета типа „моды шепчущей галереи“ В световоде с бегущей волной показателя преломления
}

\author{
() И.О. Золотовский, Д.А. Коробко, П.П. Миронов, Д.И. Семенцов, \\ А.А. Фотиади, М.С. Явтушенко
}

Научно-исследовательский технологический институт им. С.П. Капицы

Ульяновского государственного университета,

432017 Ульяновск, Россия

e-mail: rafzol.14@mail.ru

Поступила в Редакцию 01.11.2017 г.

В работе показано, что в цилиндрическом световоде с пространственно-временно́й неоднородностью, формируемой бегущей волной показателя преломления, может быть реализована сильная и сверхбыстрая модуляция туннелирующего волнового пакета типа „моды шепчущей галереи“. Показано, что соответствующие световоды могут быть использованы для эффективной генерации пико- и субпикосекундных лазерных импульсов.

DOI: $10.21883 /$ OS.2018.03.45662.259-17

\section{Введение}

Известно, что под волновым пакетом (ВП) типа моды шепчущей галереи (МШГ), как правило, подразумевается волна, распространяющаяся вдоль экватора микросферы и многократно отражающаяся от сферической границы стекло-воздух [1-4]. Подобные волны могут возбуждаться также в бочкообразных участках световолокна $[5,6]$ и в цилиндрических световодах [7]. В последнем случае они называются туннелирующими модами [8]. Подобного рода ВП распространяется внутри стеклянного световода-цилиндра по спирали с постоянным шагом. Среди особенностей туннелирующих волн типа МШГ важным является тот факт, что продольная (вдоль оси световода) групповая скорость подобного рода ВП может быть сколь угодно меньше скорости света в вакууме [9-12].

В этой связи нам представляется интересным рассмотрение динамики волнового пакета типа „туннелирующей волны“ в световодах с реализуемой бегущей волной показателя преломления (БВПП), синхронизованной с распространяющимся излучением [13-16]. Соответствующее динамическое изменение показателя преломления в световоде относительно легко может быть реализовано при возбуждении в нем акустической волны $[17,18]$, либо при размещении вдоль световода модуляторов показателя преломления [19,20].

В настоящей работе рассматривается распространение поверхностных волн, медленно туннелирующих вдоль световода. Исследуется ситуация, когда в световоде реализована бегущая волна показателя преломления (БВПП), и фазовая скорость этой волны совпадает c продольной составляющей групповой скорости поверхностной волны. Показано, что в соответствующих световодах с динамически изменяющимся показателем преломления возможна сильная модуляция и спектральное уширение ВП (при сохранении линейности чирпа) на длинах менее $10 \mathrm{c}$.

\section{Волновое поле поверхностной туннелирующей волны}

При введении света в цилиндрический волновод под некоторым углом к образующей цилиндра (рис. 1) поверхностная волна в общем случае распространяется по спиральной траектории [21]. Продольная составляющая волнового вектора такой волны $k_{z}=\left(k^{2}-k_{r}^{2}\right)^{1 / 2}$, где $k=k_{0} n(\omega), k_{0}=\omega / c, \omega$ и $c$ - частота и скорость света в вакууме, $n(\omega)$ - показатель преломления материала световода, $k_{r}$ - радиальная компонента волнового вектора. Если угол ввода волны в световод достаточно близок к нормали (по отношению к образующей), то распространение волны вдоль его оси существенно замедляется. Такие сверхмедленные волны получили название туннелирующих. Подобные волны могут быть также получены в неоднородном световоде с плавно изменяющимся диаметром. При этом должно выполняться равенство $k_{r} a=$ const, где $a$ - диаметр волновода, в общем случае зависящий от продольной координаты $z$, что дает дополнительную возможность управления параметром $k_{r}$ и как следствие параметром $k_{z}$. Так, в световоде с уменьшающимся диаметром в результате

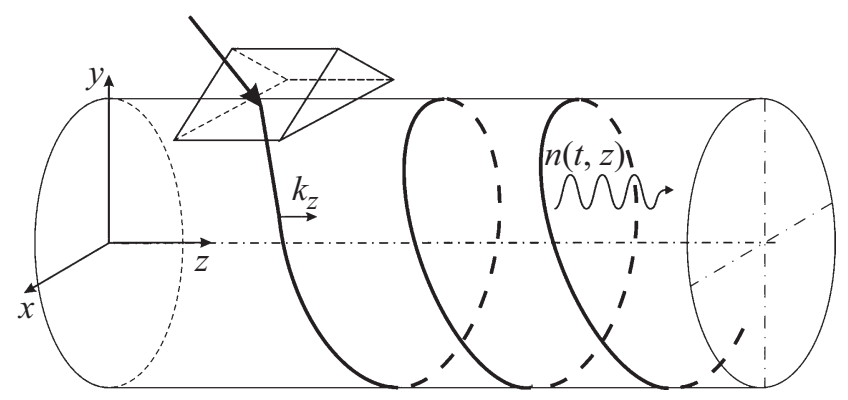

Рис. 1. Призменный ввод луча в цилиндрический световод и его траектория типа МШГ [21]. 
увеличения радиальной составляющей волнового числа можно уменьшить (вплоть до нуля) продольную составляющую групповой скорости.

В дальнейшем мы рассматриваем случай поверхностной волны, медленно туннелирующей (со скоростью $V_{z} \ll c$ ) вдоль оси $z$ (поэтому $k_{z} \ll k \sim k_{r}$ ) в световоде с изменяющимся по длине диаметром $a(z)$. В этом случае волновое электрическое поле волны можно представить в виде

$$
E(z, r, t, \varphi)=A(z, t) \Phi(r, z, \varphi) \exp \left(i \omega t-i \int_{0}^{z} k_{z}(z) d z\right),
$$

где $A(z, t)$ - медленно меняющаяся амплитуда, описывающая продольное (вдоль оси $z$ ) распределение поля туннелирующей волны, а функция $\Phi(r, z, \varphi)$ определяет радиальную и азимутальную зависимости поля в световоде. В квазилинейном приближении для однородного цилиндрического световода эту функцию при $r \leq a$ для азимутальной моды $m$-го порядка можно записать [17] в виде

$$
\Phi_{m}(r, \varphi)=\Phi_{0} \operatorname{Ai}\left[\sqrt[3]{2 m^{2}}(1-r / a)-\lambda_{m}\right] \exp (\operatorname{im} \varphi)
$$

где $\mathrm{Ai}[\ldots]$ - функция Эйри первого рода, $\lambda_{m}-$ соответствующий корень функции Эйри $[13,22], \varphi-$ угол между осью $x$ и радиусом-вектором $r$; при $r>a$ полагаем $\Phi_{m}(r, \varphi) \rightarrow 0$.

Динамика временно́й огибающей волнового пакета в таком световоде описывается уравнением

$$
\frac{\partial A}{\partial z}+V_{z}^{-1} \frac{\partial A}{\partial t}-i D_{z} \frac{\partial^{2} A}{\partial t^{2}}=G A,
$$

где введены продольная (вдоль оси $z$ ) скорость волны, дисперсия групповых скоростей (ДГС) и инкремент усиления:

$$
V_{z}(z) \simeq \gamma \frac{\partial \omega}{\partial k}, \quad D_{z}(z) \simeq \frac{1}{\gamma} \frac{\partial^{2} k}{\partial \omega^{2}}, \quad G(z) \simeq \frac{1}{\gamma}\left(g-\beta^{\prime \prime}\right) .
$$

Здесь $g$ и $\beta^{\prime \prime}$ - материальный параметр усиления и декремент затухания световода, $\gamma(z)=\left(1-k_{r}^{2} / k^{2}\right)^{1 / 2}-$ параметр продольного замедления туннелирующей волны.

Отметим, что потенциально большая площадь поверхности активного световода делает удобным создание инверсии за счет боковой накачки световода (и делает возможным использование мощных источников накачки). Это обеспечивает инверсию в большом объеме активной (усиливающей) среды и, как следствие, получение больших значений эффективного усиления $G(z)$ на единицу длины световода.

\section{Уравнения взаимодействия туннелирующего импульса с БВПП}

Будем считать, что в световоде распространяется БВПП, в которой показатель преломления среды изме- няется по закону

$$
n(t, z)=n_{0}[1-b \cos (\Omega t-q z)],
$$

где $\Omega$ - частота модуляции, $q=2 \pi / \Lambda$ - волновое число, $\Lambda$ - период, $V_{a}=\Omega / q-$ фазовая скорость БВПП, $b=\Delta n / n_{0}$ - глубина модуляции, $\Delta n-$ максимальное изменение показателя преломления. Если в световоде возбуждена акустическая волна и ее фазовая скорость $V_{a} \approx 6 \cdot 10^{3} \mathrm{~m} / \mathrm{s}$, то для синхронизации акустической и поверхностной волны параметр продольного замедления должен быть порядка величины $\gamma \approx V_{a} /(c / n)$. Для кварцевого световода со стандартным значением $n \approx 1.5$ для синхронизации туннелирующего ВП и БВПП необходимо, чтобы $\gamma \approx 3 \cdot 10^{-5}$. При этом глубина модуляции показателя преломления может достигать значений $|b|=3 \cdot 10^{-4}[22]$.

Рассмотрим однонаправленное распространение БВПП и частотно-модулированного (ЧМ) гауссова импульса с начальными параметрами [23,24]:

$$
A(t, z=0)=A_{0} \exp \left(-\left(\tau_{0}^{-2}+i C_{0}\right) t^{2} / 2\right),
$$

где $A_{0}=\sqrt{P_{0}}, P_{0}-$ мощность вводимого в световод излучения, $\tau_{0}=\tau_{u}(0)$ и $C_{0}-$ начальные длительность и скорость ЧМ (чирп) импульса. При $V_{z} \approx V_{a}$ между поверхностной „туннелирующей“ волной и БВПП происходит сильное резонансное взаимодействие. В этом случае уравнение, описывающее динамику медленно меняющейся амплитуды продольного распределения поля туннелирующей волны с учетом ее взаимодействия с БВПП, в координатах бегущего времени $\tau=t-\int_{0}^{z} d z / V_{z}(z)$ можно представить в виде

$$
\frac{\partial A}{\partial z}-i D_{z} \frac{\partial^{2} A}{\partial \tau^{2}}-G A=i k b \gamma^{-1} \cos [\Omega(\tau-\delta \tau)] A,
$$

где параметр $\delta \tau=\left(V_{a}^{-1}-V_{z}^{-1}\right) z$ характеризует временную отстройку, связанную с разностью групповой скорости импульса и скорости БВПП. Для импульсов с длительностью $\tau_{u}|\Omega| \ll 1$ и достаточно малой отстройкой $|\delta \tau| \approx\left(10^{-10}-10^{-11}\right)$ s в правой части уравнения (7) можно использовать разложение

$$
\cos [\Omega(\tau-\delta \tau)] \approx 1-\Omega^{2}(\tau-\delta \tau)^{2} / 2 .
$$

С учетом этого разложения уравнение (7) принимает вид

$$
\frac{\partial A}{\partial z}=i D_{z} \frac{\partial^{2} A}{\partial \tau^{2}}-G A=i\left(S_{1}+S_{2} \tau+S_{3} \tau^{2}\right) A,
$$

где функции $S_{j}(z)$ определены следующими выражениями:

$$
\begin{gathered}
S_{1}=b k \gamma^{-1}\left(1-\Omega^{2} \delta \tau^{2} / 2\right), \quad S_{2}=b k \gamma^{-1} \Omega^{2} \delta \tau, \\
S_{3}=-b k \gamma^{-1} \Omega^{2} / 2 .
\end{gathered}
$$

Отметим, что при $S_{2} \rightarrow 0$ возникает полная синхронизация волн, которая достижима в случае точного выбора угла ввода излучения в световод (либо подбора параметра $\gamma$ ). 


\section{Формирование локализованных ВП}

Рассмотрим подробнее динамику изначально спектрально ограниченных (т.е. $C_{0}=0$ ) импульсов типа МШГ в рассматриваемых цилиндрических световодах. Перепишем уравнение (9) в виде

$$
\frac{\partial B}{\partial z}-i D_{z} \frac{\partial^{2} B}{\partial \tau_{n}^{2}}=i S_{3} \tau_{n}^{2} B,
$$

где введены амплитуда

$$
B=A \exp \left(-i \int_{0}^{z}\left(S_{1}-S_{2}^{2} / 4 S_{3}-i G\right) d z\right)
$$

и „бегущее“ время в параболической потенциальной яме $\tau_{n}=\tau+\left(S_{2} / 2 S_{3}\right)$. Полученное уравнение хорошо известно как уравнение для гармонического осциллятоpa $[25]$.

Соответствующее уравнение при $S_{3} / D_{z}<0$ и $D_{z}<0$ имеет солитоноподобные решения типа функций гармонического осциллятора $[13,25]$ :

$$
\begin{aligned}
B\left(z, \tau_{n}\right)= & B_{0} \exp \left(-\frac{\tau_{n}^{2}}{2 \tau_{u}^{2}}\right) H_{i}\left(\tau_{n} / \tau_{u}\right) \\
& \times \exp \left(i \int_{0}^{z} \sqrt{-D_{z} S_{3}(z) / 2} d z\right),
\end{aligned}
$$

где $H_{i}(\tau)$ - полином Чебышева-Эрмита $l$-го порядка, определяемый соотношением:

$$
H_{l}(\Lambda)=\frac{(-1)^{l}}{\sqrt{\sqrt{\pi} 2^{l} l !}} \exp \left(\Lambda^{2}\right) \frac{d^{l}}{d \Lambda^{l}}\left(\exp \left(-\Lambda^{2}\right)\right),
$$

a $\Lambda=\tau_{n} / \tau_{u}$. Выражение (11) представляет собой распространяющийся вдоль оси $z$ солитоноподобный импульс с длительностью $\tau_{u} \simeq\left(-D_{z} / 2 S_{3}\right)^{1 / 4}$ и энергией

$$
W(z)=W_{0} \exp \left[2 \int_{0}^{z} G(z) d z\right]
$$

где $W_{0}$ - энергия вводимого в световод ВП. Отметим, что в рассматриваемом случае солитоноподобный импульс может быть получен и в среде с нормальной дисперсией, т.е. при $D_{z}>0$. Соответствующий режим реализуется в случае $S_{3}<0$ (при $b>0$ ), что соответствует синхронизации ВП с минимумом в зависимости $n(t, z)$ в БВПП. Данное обстоятельство делает подобного рода ВП более устойчивым по отношению к влиянию модуляционной неустойчивости [23,24].

\section{Сверхсильная модуляция поверхностного ВП}

Практический интерес представляет такой режим динамики ВП, при котором $S_{3} / D_{z}>0$. Рассмотрим динамику изначально частотно-модулированного ВП, для которого начальный чирп $C_{0}$ отличен от нуля. В этом случае амплитуду огибающей ВП можно представить в виде

$$
A(z)=B(z) \exp \left[i\left(\xi(z) \tau+\alpha(z) \tau^{2}\right)\right]
$$

где $\xi$ - изменение в поле БВПП скорости максимума огибающей, вызванное расстройкой групповой скорости частотно-модулированной волны и скорости БВПП, $\alpha$ - скорость наведенной БВПП частотной модуляции. Подставим выражение (14) в уравнение (7). В результате для введенных параметров приходим к следующей системе уравнений:

$$
\begin{gathered}
\frac{\partial B}{\partial z}+2 D_{z}(\xi+2 \alpha \tau) \frac{\partial B}{\partial \tau}-i D_{z} \frac{\partial^{2} B}{\partial \tau^{2}} \\
=i\left(S_{1}-\xi^{2} D_{z}+2 i \alpha D_{z}\right) B, \\
\frac{\partial \xi}{\partial z}+4 \alpha D_{z} \xi=S_{2}, \\
\frac{\partial \alpha}{\partial z}+4 D_{z} \alpha^{2}=S_{3} .
\end{gathered}
$$

Далее с помощью подстановки

$$
B=\bar{B} \exp \left[i \int_{0}^{z}\left(S_{1}-\xi^{2} D_{z}+2 i \alpha D_{z}\right) d z\right]
$$

уравнение (15a) преобразуется к виду

$$
\frac{\partial \bar{B}}{\partial z}-i D_{z}(z) f^{2}(z) \frac{\partial^{2} \bar{B}}{\partial \tau^{\prime 2}}=0
$$

где введены параметр

$$
f(z)=\exp \left(-4 \int_{0}^{z} D_{z}(z) \alpha(z) d z\right)
$$

и нормированное бегущее время

$$
\tau^{\prime}(z) \equiv f(z) \tau-2 \int_{0}^{z} f(z) D_{z}(z) \xi(z) d z .
$$

Решение уравнения (17) с начальным условием (6) можно представить в виде

$$
\bar{B}\left(\tau^{\prime}, z\right)=A_{0} F^{-1 / 2}(z) \exp \left[-\frac{\left(\tau_{0}^{-2}+i C_{0}\right) \tau^{\prime 2}}{2 F(z)}\right],
$$

где введены обозначения

$F(z)=1-2 g(z)\left(C_{0}-i \tau_{0}^{-2}\right), \quad g(z)=\int_{0}^{z} D_{z}(z) f^{2}(z) d z$.

В предположении $\xi=0$ и $S_{2}=0$ (когда групповая скорость ВП и скорость БВПП равны и, как следствие, 
$\delta \tau=0)$ получаем выражения для длительности ЧМ импульса:

$$
\tau_{u}(z)=\frac{\tau_{0}}{f(z)} \sqrt{\left(1-2 C_{0} g(z)\right)^{2}+\frac{4 g^{2}(z)}{\tau_{0}^{4}}},
$$

и его реального чирпа:

$$
C_{\mathrm{ef}}(z)=\bar{C}(z)-2 \alpha(z)
$$

где величина

$$
\bar{C}(z)=\frac{C_{0}-2\left(C_{0}^{2}+\tau_{0}^{-4}\right) g(z)}{\left(1-2 C_{0} g(z)\right)^{2}+4 \tau_{0}^{-4} g^{2}(z)} .
$$

Скорость наведенной БВПП частотной модуляции $\alpha(z)$ в выражении (22) может быть найдена из решения уравнения (15в). Для случая $D_{z} S_{3}>0$ получаем

$$
\alpha= \pm \sqrt{\frac{S_{3}}{4 D_{z}}} \operatorname{th}\left(2 \sqrt{S_{3} D_{z}} z\right)
$$

где знак „+““ соответствует $S_{3}>0$ и синхронизации импульса с максимумом показателя преломления в БВПП при нормальной материальной дисперсии световода $D_{z}>0$, а знак „-“ “ соответствует $S_{3}<0$ и синхронизации импульса с минимумом показателя преломления в БВПП при аномальной материальной дисперсии световода $D_{z}<0$.

Полученные решения показывают, что при взаимодействии „медленного“ ВП с БВПП возможна его сверхбыстрая модуляция при сохранении линейности чирпа и большой ширине спектра. Это в свою очередь позволяет обеспечить дальнейшее сжатие импульса. Так, при положительном значении чирпа сжатие можно осуществить на дифракционных решетках. Если же у импульса сформировался отрицательный чирп, то для его дальнейшего сжатия может быть использован обычный световод с нормальной материальной дисперсией. В обоих случаях длительность импульса после компрессии (когда импульс становится спектрально ограниченным c $\left.C_{\text {ef }} \rightarrow 0\right)$ определяется соотношением [15,23]:

$$
\tau_{\min }=\tau_{u}(z) / \sqrt{1+C_{\mathrm{ef}}^{2}(z) \tau_{u}^{4}(z)} \cong 1 / 2|\alpha(z)| \tau_{u}^{2}(z) .
$$

Из приведенных соотношений следует, что при исходной длительности вводимого в световод-модулятор спектрально ограниченного $\left(C_{0}=0\right.$ и $\left.\alpha_{0}=0\right)$ импульса c $\tau_{0}=10^{-11} \mathrm{~s}$, глубиной и частотой модуляцией БВПП $b k \sim \pm 2 \cdot 10^{2} \mathrm{~m}^{-1}, \Omega \sim 3 \cdot 10^{9} \mathrm{~s}^{-1}, \gamma \sim 10^{-5}$ и эффективной ДГС $\left|D_{z}\right|=\left(10^{-21}-10^{-22}\right) \mathrm{s}^{2} / \mathrm{m}$ скорость частотномодулированного импульса на длине цилиндрического световода-модулятора $z \sim 1$ см составит $\left|C_{\mathrm{ef}}\right| \approx 10^{24} \mathrm{~s}^{-2}$. При этом его длительность существенным образом не изменится. В результате, после пропускания импульса через диспергирующий элемент, обеспечивающий временную компрессию, длительность импульса может уменьшиться в 100 раз до значения $\tau_{u}=10^{-13} \mathrm{~s}$.

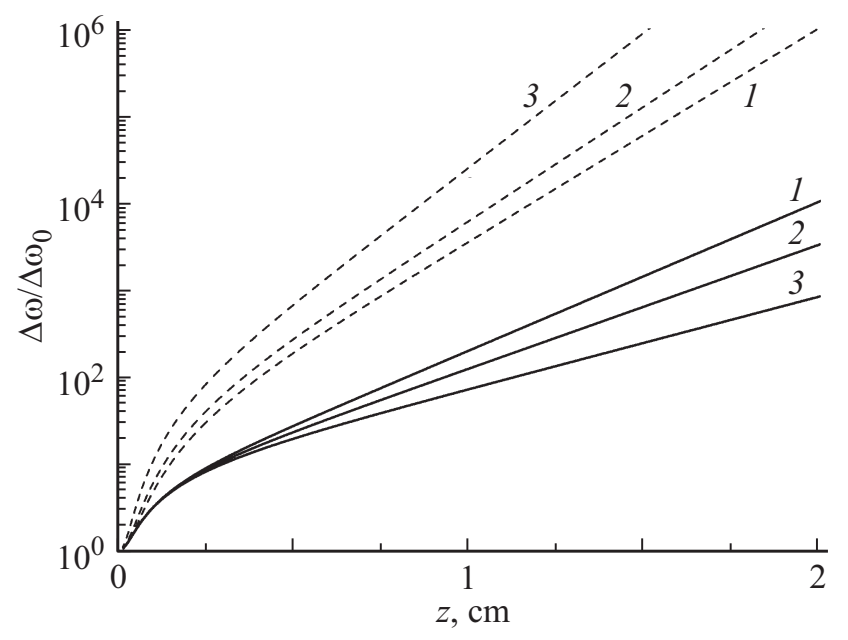

Рис. 2. Зависимость нормированной ширины спектра от длины световода при $b=-3 \cdot 10^{-4}$ и $D_{z}=$ $=(0.5,0.9,1.3) \cdot 10^{-21} \mathrm{~s}^{2} / \mathrm{m} \quad$ (соответственно сплошные кривые $1-3)$; при $D_{z}=1 \cdot 10^{-21} \mathrm{~s}^{2} / \mathrm{m} \quad$ и $b=(-2,-2.3,-3.2) \cdot 10^{-4}$ (соответственно штриховые кривые 1-3).

Так как длительность импульса связана с его спектральной шириной соотношением $\tau_{\min } \simeq 1 / \Delta \omega(z)$, то для ширины спектра соответствующего ВП получаем выражение

$$
\Delta \omega(z)=\sqrt{\tau_{u}^{-2}(z)+C_{\mathrm{ef}}^{2}(z) \tau_{u}^{2}(z)} .
$$

Для рассматриваемых значений используемых параметров с высокой степенью точности $|\bar{C}(z)| \ll|\alpha(z)|$, поэтому выражение (26) можно представить в виде:

$$
\Delta \omega=\sqrt{\tau_{u}^{-2}+4 \alpha^{2} \tau_{u}^{2}} .
$$

На рис. 2, $b$ представлены зависимости нормированной ширины спектра импульса $\Delta \omega$ от проходимого по световоду пути $z$ при следующих значениях параметров световода, ВП и БВПП: $\tau_{0}=10^{-11} \mathrm{~s}, k=6 \cdot 10^{6} \mathrm{~m}^{-1}$, $\gamma=3 \cdot 10^{-5}, \Omega=10^{9} \mathrm{~s}^{-1}, \Delta \omega_{0}=10^{11} \mathrm{~s}^{-1}$. Сплошные линии отвечают глубине модуляции $b=-3 \cdot 10^{-4}$ и дисперсии $D_{z}=(0.5,0.9,1.3) \cdot 10^{-21} \mathrm{~s}^{2} / \mathrm{m}$ (кривые $1-3)$, штриховые - $D_{z}=10^{-21} \mathrm{~s}^{2} / \mathrm{m}$ и $b=$ $=(-2,-2.3,-3.2) \cdot 10^{-4}$ (кривые $\left.1-3\right)$. Видно, что при выбранных параметрах значительного уширения спектра (в 100 раз и более) можно достичь на длинах световода менее $1 \mathrm{~cm}$.

На рис. 3 показаны зависимости наведенного БВПП чирпа от длины световода при тех же параметрах, что и на рис. 2. Из рис. 3 следует, что чирп в зависимости от пройденного пути нарастает линейно и достигает насыщения.

На рис. 4 показана зависимость чирпа импульса от глубины модуляции $b$ при $z=1 \mathrm{~cm}$ при тех же значениях параметров, что и на предыдущих рисунках, и отвечающая дисперсии $D_{z}=(0.5,0.9,1.3) \cdot 10^{-21} \mathrm{~s}^{2} / \mathrm{m}$ 


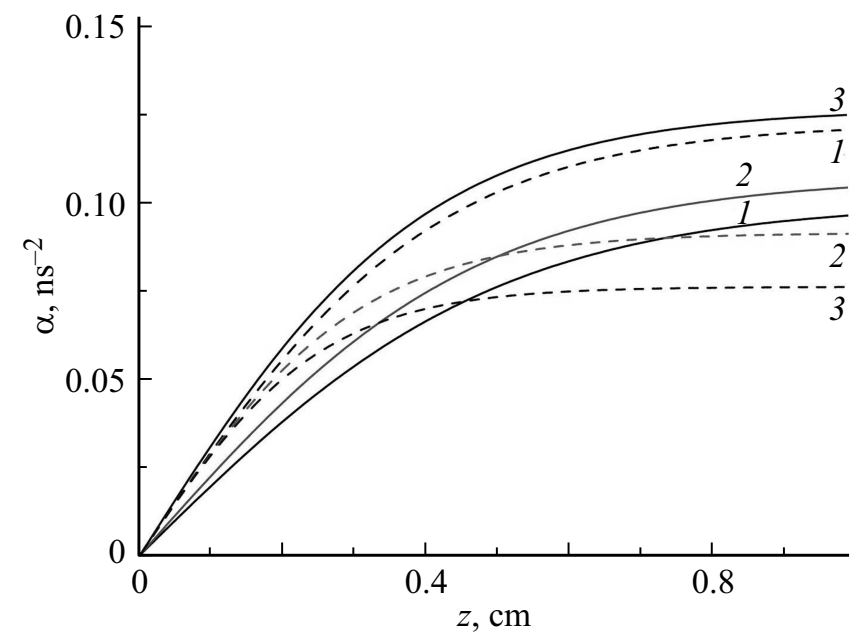

Рис. 3. Зависимость наведенного БВПП чирпа от длины световода при $b=-3 \cdot 10^{-4}$ и $D_{z}=(0.5,0.9,1.3) \cdot 10^{-21} \mathrm{~s}^{2} / \mathrm{m}($ coответственно сплошные кривые $1-3)$; при $D_{z}=1 \cdot 10^{-21} \mathrm{~s}^{2} / \mathrm{m}$ и $b=(-2,-2.3,-3.2) \cdot 10^{-4}$ (соответственно штриховые кривые $1-3)$.

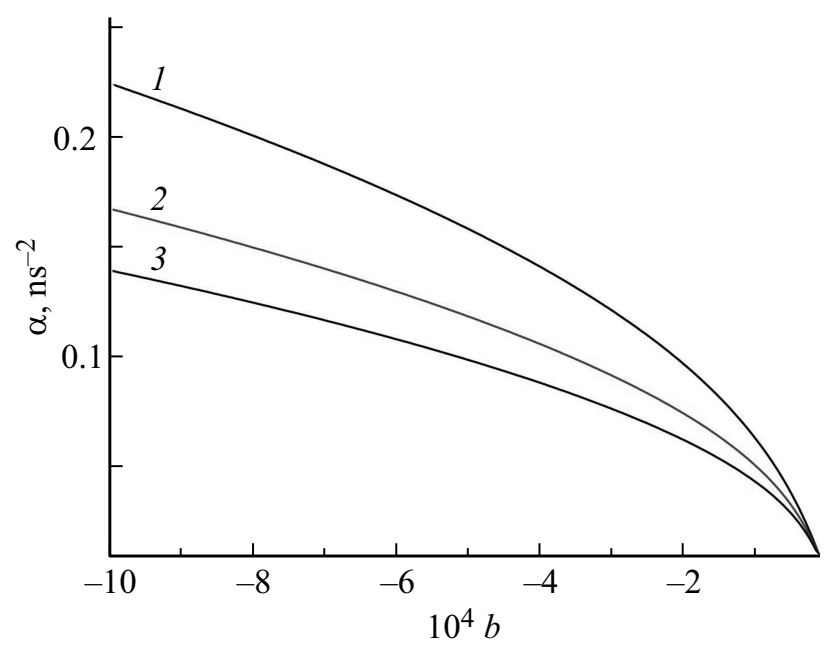

Рис. 4. Зависимость чирпа импульса от глубины модуляции $b$ при $z=1 \mathrm{~cm}$ для $D_{z}=(0.5,0.9,1.3) \cdot 10^{-21} \mathrm{~s}^{2} / \mathrm{m}$ (соответственно кривые $1-3)$.

(кривые 1-3). Из рис. 4 следует, что значение чирпа растет с увеличением модуля глубины модуляции $b$.

Если импульс изначально синхронизован с минимумом волны показателя преломления (когда $b>0$ ), то дальнейшее сжатие импульса должно осуществляться в среде с нормальной материальной дисперсией. В этом случае в качестве компрессора может быть использован обычный световод с нормальной дисперсией. Если же импульс синхронизован с максимумом БВПП $(b<0)$, то сжатие импульса должно осуществляться в среде с аномальной дисперсией. В этом случае, если пиковая мощность импульса меньше $100 \mathrm{~W}$, то в качестве компрессора может также использоваться обычный волоконный световод, но с аномальной дисперсией. Если же пиковая мощность значительно больше $1 \mathrm{~kW}$ (что может представлять особый интерес), то для нивелирования нежелательных нелинейных эффектов предпочтительно использование пары дифракционных решеток.

\section{Заключение}

Проведенный в работе анализ показывает, что световоды с реализованной в них БВПП, синхронизованной по скорости распространения с вводимым в световод импульсом с малой (много меньше скорости света в вакууме) продольной скоростью, могут быть использованы для его сильной частотной модуляции на малой длине (значительно меньше $10 \mathrm{~cm}$ ) световода-модулятора. В этом случае уже на этой длине световода можно добиться значительного спектрального уширения импульса (на 1-3 порядка - до значений $\Delta \omega \cong 10^{14} \mathrm{~s}^{-1}$ ) при практически идеальном сохранении линейности чирпа (т.е. при квадратичной зависимости фазы от времени). Последнее обстоятельство в свою очередь делает возможным последующее сильное сжатие (временную компрессию) импульса (на 1-3 порядка) вплоть до субпикосекундных и фемтосекундных длительностей в оптическом диапазоне излучения. С другой стороны, соответствующие световоды- модуляторы (как в волоконном, так и планарном исполнении) могут быть использованы для спектрального сжатия (спектральной компрессии) исходно частотно-модулированных широкополосных импульсов.

Работа выполнена при поддержке Министерства образования и науки РФ (в рамках задания № 3.3889.2017. ПЧ и проекта № 14.Z50.31.0015 Минобрнауки РФ и проекта РНФ № 16-42-02012).

\section{Список литературы}

[1] Брагинский В.Б., Ильченко В.С., Городецкий М.Л. // УФН. 1990. T. 160. № 1. C. 157.

[2] Frateschi N.C., Levi A.F.J. // Appl. Phys. Lett. 1995. V. 66. P. 2932.

[3] Wang R.P., Dumitrescu M. // J. Appl. Phys. 1997. V. 81. P. 3391.

[4] Murugan G.S., Panitchob Y., Tull E.J., Bartlett P.N., Howak D.W., Zervas M.N., Wilkinson J.S. // J. Appl. Phys. 2010. V. 107. P. 053105.

[5] Sumetsky M. // Opt. Lett. 2004. V. 29. N 1. P. 8.

[6] Suchkov S.V., Sumetsky M., Sukhorukov A.A. // Opt. Lett. 2015. V. 40. P. 3806.

[7] Suchkov S.V., Sumetsky M., Sukhorukov A.A. // Opt. Lett. 2017. V. 42. P. 2149.

[8] Sumetsky M. // Opt. Express. 2012. V. 20. P. 22537.

[9] Sumetsky M., Dulashko Y. // Opt. Express. 2012. V. 20. P. 27896.

[10] Murugan G.S., Wilkinson J.S., Zervas M.N. // Opt. Express. 2009. V. 17. P. 11916

[11] Sumetsky M., Tong L. // Opt. Commun. 2012. V. 285. N 23. P. 4639. 
[12] Dvoyrin V., Sumetsky M. // Opt. Lett. 2016. V. 41. P. 5547.

[13] Zhuravlev V.M., Zolotovskii I.O., Mironov P.P. // Opt. Spectr. 2016. V. 121. N 5. P. 744.

[14] Zolotovskii I.O., Korobko D.A., Lapin V.A., Sementsov D.I. // Opt. Spectr. 2016. V. 121. N 2. P. 256.

[15] Золотовский И.О., Лапин В.А., Семениов Д.И. // Квант. электрон. 2016. T. 46. № 1. C. 39. Zolotovskii I.O., Lapin V.A., Sementsov D.I. // Quant. Electron. 2016. V. 46. N 1. P. 39.

[16] Торчигин В.П. // Квант. электрон. 1995. Т. 22. № 5. С. 509. Torchigin V.P. // Quant. Electron.1995. V. 25. N 5. P. 484.

[17] Торчигин В.П. // ЖТФ. 1996. Т. 64 № 4. С. 128.

[18] Торчигин В.П. // Квант. электрон. 1993. Т. 20. № 3. С. 276. Torchigin V.P. // Quant. Electron. 1993. V. 23. N 3. P. 235.

[19] Goutzoulis A.P., Pape D.R. Design and fabrication of acoustooptic devices. N.Y.: Marcel Dekker, 1994. 497 p.

[20] Торчигин В.П. // Квант. электрон. 1993. Т. 20. № 3. С. 283. Torchigin V.P. // Quant. Electron. 1993. V. 23. N 3. P. 241.

[21] Сычугов В.А., Магдич Л.Н., Торчигин В.П. // Квант. электрон. 2001. T. 31. № 12. С. 1089. Sychugov V.A., Magdich L.N., Torchigin V.P. // Quant. Electron. 2001. V. 31. N 12. P. 1089.

[22] Бондаренко В.С., Зоренко В.П., Чкалова В.В. / Акустооптические модуляторы света М.: Радио и связь, 1988. С. 134.

[23] Ахманов С.А., Выслоух В.А., Чиркин А.С. Оптика фемтосекундных лазерных импульсов. М.: Наука, 1988. 312 с.

[24] Кивщарь Ю.С., Агравал Г.П. Оптические солитоны. М.: Физматлит, 2005. 648 с.

[25] Ландау Л.Д., Ливщии Е.М. Квантовая механика. М.: Наука, 1989. $767 \mathrm{c}$. 